\title{
Application of Combined Mean Atomic Number Backgrounds and Blank Corrections to EPMA Trace Element Measurements: Successes and Challenges
}

Owen K. Neill ${ }^{1}$, Nikita L. La Cruz ${ }^{1}$, David Hernández Uribe ${ }^{2}$, Jameson S. R. Jolles ${ }^{1}$, Joseph R. Boro ${ }^{3}$, and John J. Donovan ${ }^{4}$

1. School of Earth and Environmental Sciences, University of Michigan, Ann Arbor, MI, USA.

2. Department of Geology and Geological Engineering, Colorado School of Mines, Golden, CO, USA.

3. School of the Environment, Washington State University, Pullman, WA, USA

4. CAMCOR, University of Oregon, Eugene, OR, USA.

*Corresponding author: okneill@umich.edu

The measurement of trace elements by electron microprobe can be both difficult and time consuming, as extended counting times are often necessary to obtain acceptable precision at low concentrations. Furthermore, while generally insignifcant when measuring major elements, small accuracy errors in continuum measurements can cause systematic over- or underestimates of concentration. A recently developed method [1] deals with both of these issues. This method combines the use of mean atomic number (MAN) background measurements [2], based on Kramers' law [3] relating the mean atomic number $(\bar{Z})$ of a sample to the intensity of the continuum produced at a given electron dose, with a "blank" correction [4] that adjusts measured concentrations for minor errors in continuum measurement that will yield measurements that are systematically too high or too low. This method has the advantage of high accuracy in significantly reduced times, as the MAN method obviates the need for direct offpeak measurements of continuum, and the blank correction eliminates the small errors in measured continuum that result accuracy errors.

The application of this method to trace elements in simple matrices has, in general, yielded excellent results, not only in terms of precision, accuracy, and sensitivity, but also in terms of efficiency. Measurements of rutile using this method yield highly accurate results for $\mathrm{Cr}, \mathrm{Fe}, \mathrm{Nb}$ and $\mathrm{Zr}$, while also decreasing analysis times by over $50 \%$ per point relative to off-peak measurements (Fig. 1). Similarly, measurements of $\mathrm{Ti}$ in quartz, a commonly used thermometer, can be obtained with detection limits of $<5 \mu \mathrm{g} / \mathrm{g}$ in $<6$ minutes per point, with excellent accuracy (Fig. 2), if counts are aggregated from multiple spectrometers and care is taken to assure uniform conductive coatings between standards and unknowns.

However, spurious results can still be obtained using the combined MAN/blank method if the MAN curve is poorly constrained due to lack of appropriate standards (which may be especially problematic with high $\bar{Z}$ materials). Also, if the matrix of the blank standard does not match the unknown sample, the shape and intensity of the continuum may also change, and render the "blank" measurement inaccurate, leading to significant over-estimates or under-estimates of "blank" level, and therefore significant inaccuracies in measured concentration. This can be seen in the case of measurements of Au-Ag alloys, where the matrix varies considerably (Fig. 3), unlike in measurements of quartz or rutile, where the matrix is essentially identical to the blank standards. Furthermore, the effects of using a blank correction in situations where X-ray spectral interferences are significant are still to be investigated.

To summarize, the combined MAN-blank method must be deployed carefully to avoid spurious results, but when used appropriately, it can yield precise, highly accurate trace element measurements in a fraction of the time required by traditional off-peak background measurements. 


\section{References:}

[1] JJ Donovan, JW Singer and JT Armstrong, Am Mineral 101 (2016), p. 1839-1853.

[2] JJ Donovan and TN Tingle, J Microsc Microanal, 8 (1996), p. 1-7.

[3] HA Kramers, Phil Mag, 46 (1923), p. 836.

[4] JJ Donovan, HA Lowers and BG Rusk, Am Mineral 96(2-3) (2011), p. 274-282.
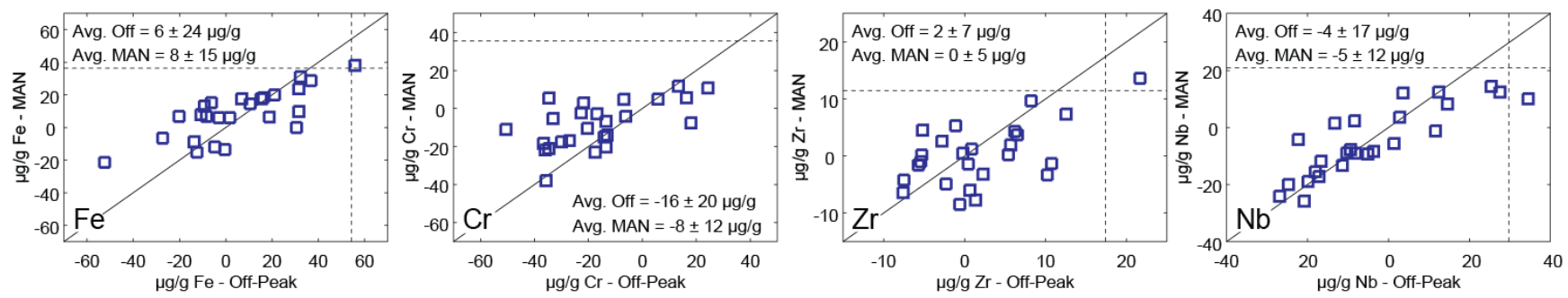

Figure 1. Measurements of trace elements in trace element free synthetic rutile using the combined MAN-blank method, using a separate synthetic rutile as a blank standard. Dashed lines represent statistical lower limits of detection; solid lines represent 1-1 correlation.

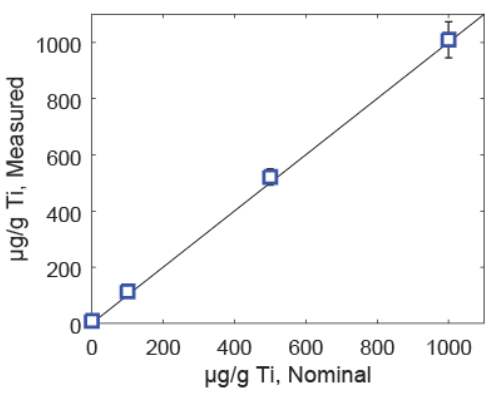

Figure 2. Measurements of Ti-doped quartz standards from the University of Edinburgh using the combined MAN-blank method, with Herkimer quartz used as a blank standard. Solid line represents 1-1 correlation.
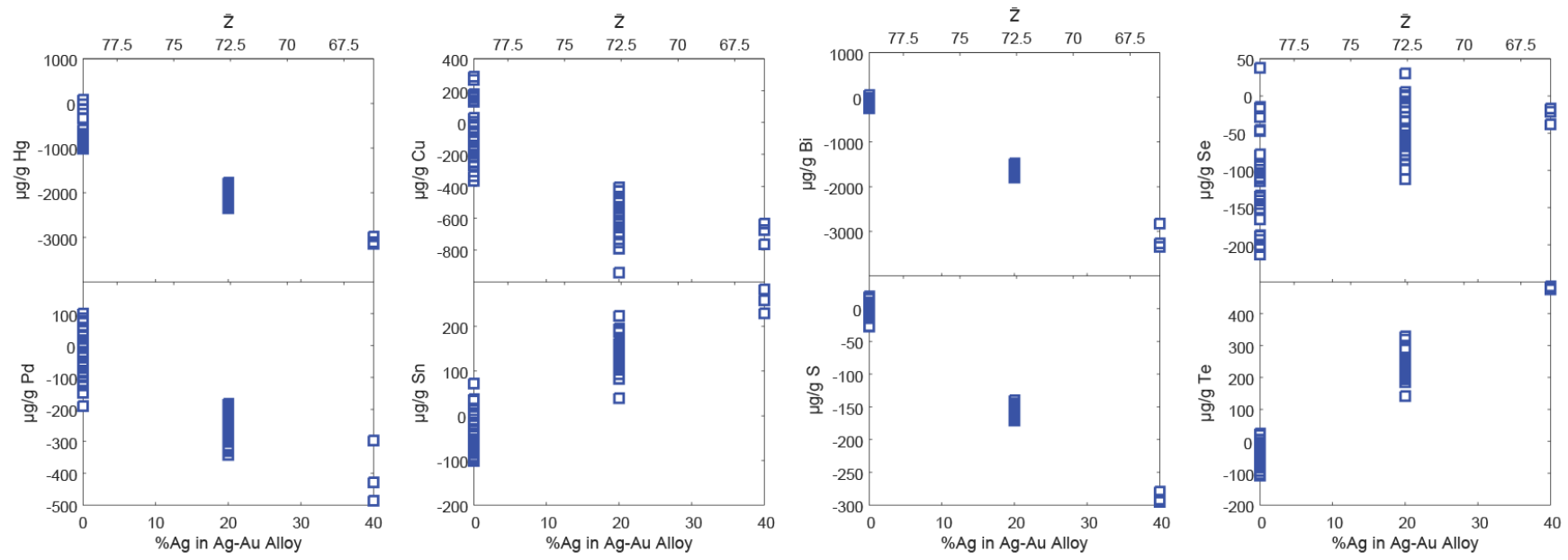

Figure 3. Measurements of trace elements in Au-Ag alloys using the combined MAN-blank method, with NIST SRM 481 pure gold used as a blank standard. 